\title{
Single Particle Analysis with the Volta Phase Plate
}

\author{
Radostin Danev ${ }^{1}$, Maryam Khoshouei ${ }^{1}$, Wolfgang Baumeister ${ }^{1}$ \\ 1. Max Planck Institute of Biochemistry, Martinsried, Germany.
}

Cryo-EM single particle analysis is experiencing a "renaissance" brought about by the development and commercialization of direct electron detectors. In the last few years the typical performance shifted from sub-nanometer $(6 \sim 10 \AA)$ to near-atomic $(<4 \AA)$ resolutions. This dramatic improvement put cryo-EM in the spotlight and piqued the interest of structural biologists from other fields, such as $\mathrm{x}$-ray crystallography. The higher signal-to-noise ratio provided by direct detectors improved not only the resolution but also the ability to solve the structures of smaller $(<300 \mathrm{kDa})$ particles $[1,2]$. However, such particles, although more accessible now, still remain challenging and require the acquisition of big datasets of thousand or more micrographs. Phase plates are one of the technologies holding promise for future improvements in single particle cryo-EM performance, especially for challenging specimens in terms of size, flexibility and heterogeneity.

Phase plates for transmission electron microscopy are devices that improve the image contrast by increasing the information transfer for low spatial frequencies. They also enable in-focus phase contrast meaning that weak phase objects, such as ice-embedded biological samples, can be observed without defocusing the objective lens. Phase plates have been in development for more than 15 years [3] but so far have seen very few applications because of practical issues related to performance instability and difficulty of use. The recent development of the Volta phase plate (VPP) [4] solved most of those issues and for the first time enabled automated data acquisition with a phase plate.

The VPP is a remarkably simple device comprising of a thin $(\sim 10 \mathrm{~nm})$ amorphous carbon film positioned at the back focal plane of the objective lens. The film is continuously heated to about $250^{\circ} \mathrm{C}$ to prevent beam-induced contamination and enable the Volta potential mode of operation. The strong central diffraction beam of unscattered electrons interacts with the film and modifies its surface properties. This creates a Volta potential difference (the contact or outer potential just above the film surface) between the spot of the central diffraction beam and the surrounding areas. This in turn leads to a three-dimensional electrostatic potential distribution above and below the film which when integrated along the beam path leads to a phase shift difference between the central diffraction beam and scattered beams. The effect of the central diffraction beam on the film is cumulative leading to a phase shift which increases with the electron dose. The increase, however, is not linear and slows down as the phase shift approaches $\sim \pi / 2$ [4], which coincidently is the ideal phase shift for a phase plate. In practice the nonconstant phase shift of the VPP is not a problem because improved image contrast is produced by a wide range of phase shifts, from $\sim \pi / 4$ to $\sim 3 \pi / 4$. Thus, many images $(\sim 50)$ can be acquired on a single spot of the VPP film before the phase shift becomes too large $(>3 \pi / 4)$ and the phase plate has to be moved to a new spot.

In our lab the VPP is used routinely in cryo-tomography applications where it has already demonstrated remarkable improvements in contrast and visibility of features [5,6]. Single particle applications of the VPP are still in their early days, but the first results are very encouraging. The improved contrast was instrumental in the near-atomic cryo-EM structure determination of the small $(\sim 250 \mathrm{kDa})$ protein complex peroxiredoxin-3 [7]. The VPP has the potential to extend the applicability of cryo-EM towards 
smaller particles and it is not unreasonable to expect that in near future even structures of under $100 \mathrm{kDa}$ could be solved to near-atomic resolutions.

In this paper we discuss the optimal experiment parameters for single particle data acquisition with the VPP. We will share our experiences, present application examples (Fig. 1) and discuss future directions.

[1] Xc Bai et al, Nature 525 (2015) p. 212.

[2] B Liang et al, Cell 162 (2015) p. 314.

[3] R Danev and K Nagayama, Ultramicroscopy 88 (2001) p. 243.

[4] R Danev et al, PNAS 111 (2014) p. 15635.

[5] S Asano et al, Science 347 (2015) p. 439.

[6] J Mahammid et al, Science in press.

[7] M Khoshouei et al, Nature Communications 7 (2016) a.n. 10534.

(a)

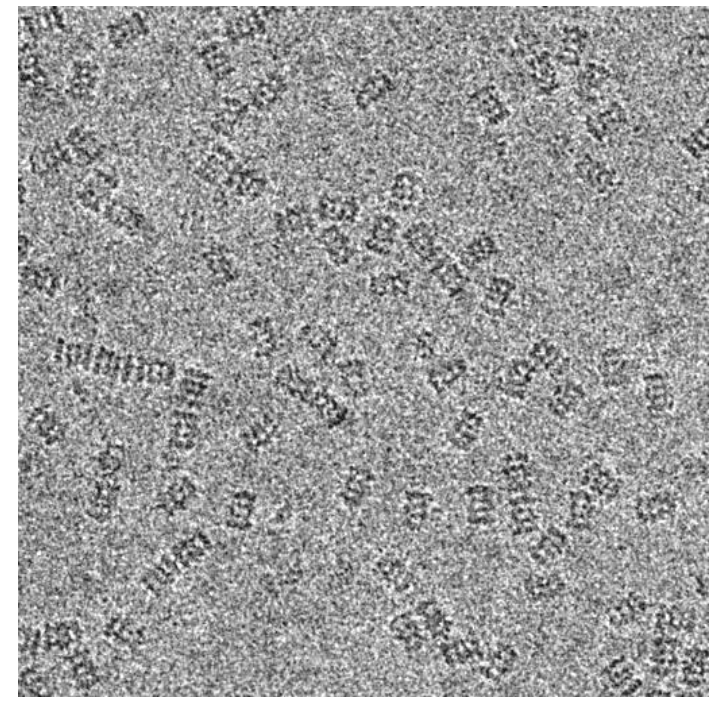

(c)

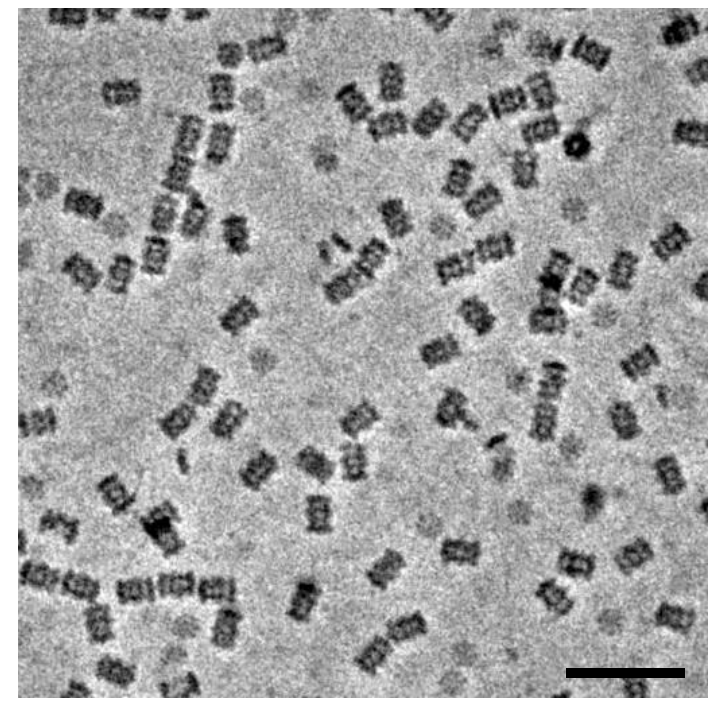

(b)

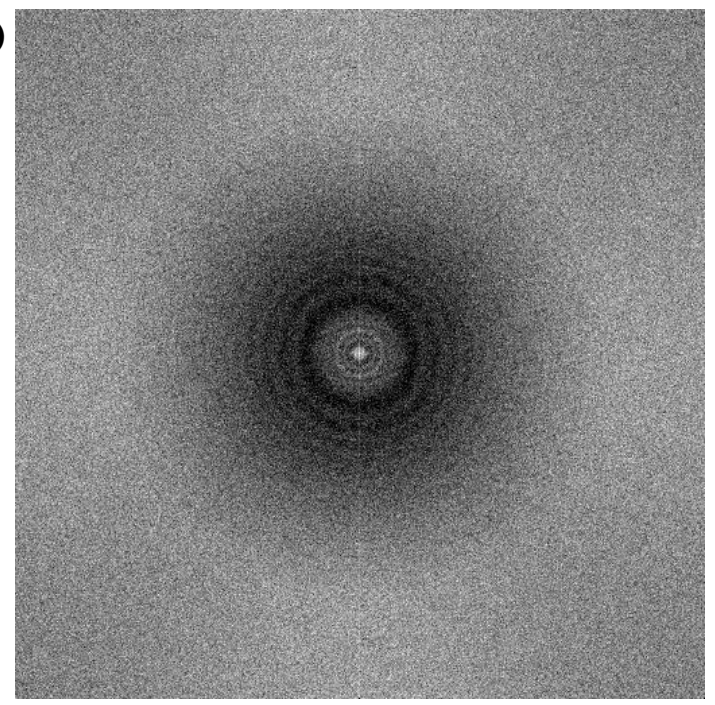

(d)

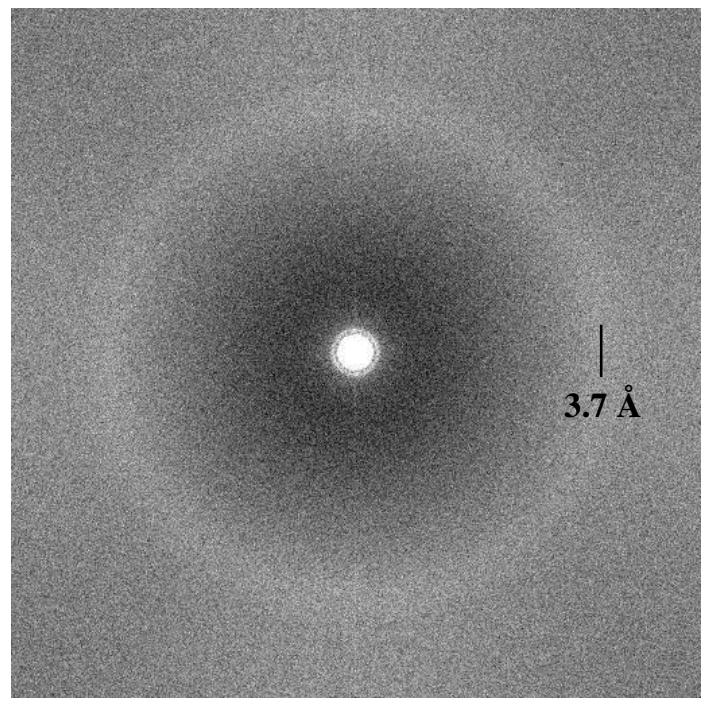

Figure 1. Cryo-EM single particle images of $20 \mathrm{~S}$ proteasome. (a) Conventional $-1.5 \mu \mathrm{m}$ defocus phase contrast image of ice-embedded 20S particles. (b) Power spectrum of the image in (a). (c) VPP in-focus image of the same sample as in (a). (d) Power spectrum of the image in (c). Scale bar: $50 \mathrm{~nm}$. 